\title{
Contrastive analysis of lexical chunks in computer English textbooks and College English textbooks
}

\author{
$\mathrm{XU}$ Yiqun ${ }^{1, \text { a }}$ \\ ${ }^{1}$ Jilin Business and Technology College, Changchun 130000,China \\ aemail: xuyiqun@163.com
}

Keywords: College English writing; chunks; reading; application

\begin{abstract}
In English learning, the lexical chunks or phrases which are composed of the lexical chunks are the significant help to the organizational memory. In College English learning, the lexical chunks can speed up the accumulation of words, while the lexical chunks can also reduce the error of context. This is for the English writing, in fact, it provides a better language environment and writing fluency. In this paper, combined with the demand of College English writing application and the application of lexical chunks and register analysis, indicating that the role of lexical chunks in English writing, the accumulation of chunks, and summarizes the application of chunks in writing notes.
\end{abstract}

\section{Introduction}

There are great differences in the habit of grammar in Chinese language and English language, many English grammar theory to Chinese grammar theory to understand if there will be a great deviation, which is an important obstacle in foreign language learning. In English learning, memorizing vocabulary is the foundation, and skilled session, writing ability is in basic words, to be the correct syntax and semantics together, rather than simply by separate lexical meaning of the constitution[1].

Has the meaning of word formation many grammatical chunks have practical significance (meaningful chunks), and then by these chunks form a continuous coherent text (continuous coherent text). University level English learning memory and comprehension is the most basic and most parts of the simple, but due to differences in language environment, when the students in English writing practice, appear in the mind think of first is usually Chinese or is based on Chinese language habits incoherent English words, which in students' writing, produce great obstacles.

Simply speaking, English language is some common English phrases in Chinese idioms or similar local vocabulary. In English, there are chunks and symmetrical, syllable beautiful rhythm coordination etc.. Chunks can be divided into free chunks, semi free chunks and fixed chunks according to the structure of the structure. Free chunks are broken chunks, which are temporary and loose. The free language block has low cohesion, its combination and aggregation. With peculiar to the English article use, for example, the article itself does not have practical significance only to that number or specified. Actually, this will article and noun polymerization,, for example a happy girl, which can be added to the very, embodies the combination of, can also replace the nouns, manifests for the polymerization. Semi - free chunks have partial or limited decomposition. A fixed position can be added or a component can be replaced. For example, in a fluster (Huang Zhang) in a fixed position to join complete, take one s breath 'away, one' s can be replaced. Fixed chunk chunk is not biodegradable, adhesion is very high, is not easy to break down, can not replace or add other ingredients. For example, go ape, hit the ceiling (crazy) (angry). Because the free chunk combination is very strong, a number of words it is not certain. Half free chunks and fixed chunks according to the number of words, words can be divided into two blocks, three words, four words block block and multi word chunks (more than four words). For example, in case, in the situation, give me a laugh, bring sb to the verge of tears[2]. 


\section{The role of lexical chunks in English Writing}

Combined with chunks of simple introduction, chunk nature is English habitual vocabulary combination form, has the unique significance and chunks of read up with specific coherent and in line with the language habits of English linguistic minorities, for example in Chinese dialects, people used to this argument, so that the course, said it is also more fluently, which contribute to an individual's memory, and the memory directly contains more than one word, on the one hand, is conducive to enhance memory, on the other hand can also enable users to more easily remember. Scholars have pointed out that the National English language learning English belongs to learn (learn, remember), and non English language national learning English belongs to the study (Study), study is time-consuming, laborious, and in English speaking countries, English is just a kind of habit, they for the language learning process is from will say will write - understand the usage of grammar, and learn our way is from the write (mainly refers to the vocabulary of writing and non coherence writing sentences and paragraphs) - understand grammar usage - says.

It would appear, the traditional Chinese way of learning English is actually "stupid", which also leads to students in English writing, on the memory of English extraction ability is poor, and children in English speaking countries in the face of writing, mind first appeared in the habit of saying (or the equivalent in chunks), and then consider the word writing. This way is similar to our primary school, junior high school language learning, writing only need to recall a single vocabulary can be successfully completed simple writing[3].

From a theoretical point of view, the United States educator Lewis.M proposed that the language block is a word fixed with a combination of fixed meaning, can be the output of language is more convenient. And some scholars have suggested that non-native speakers of other languages, the acquisition of vocabulary even more than the native state of the people, but the use of the general ability. Under normal circumstances, mother tongue learning commonly used word in 4000 can be fluent in speaking and writing, rather than mother tongue learning memory vocabulary may be beyond the range (such as our English level 4 requirements English vocabulary in 4000-4500, six in the 5000-6000). This shows the importance of language habits in language learning, language habits are popular is a feeling, and the language is an important way to form a sense of language.

The most direct effect of language chunks on English learning is to help improve the accumulation of vocabulary, to form a fluent language expression ability, and to grasp the meaning and coherence of the language. For English writing, it has more practical effect. In the previous paper, the application of lexical chunks has strengthened the sense of the language. In practical writing, the requirements of the language is slightly less than that of the conversation, but more attention is paid to the cohesion of the statement. Chunks at this time play a very important organizational skills, such as college English writing often practice standards, such as Syrian thang, description and argumentative also have their conventional word explicit marking of, chunks of accumulation to accelerated write authors memories of specific style and structure, the writing time recall of words for ratio is reduced and speed up your writing.

\section{Suggestions on the application of the lexical chunks in College English Writing}

First of all, it should be summarized, sorted and accumulated as much as possible through reading and practice. The lexical chunks are not complete. In different learners' view, the different ways of dividing the chunks may be different. For example, when learning English, some common syntax (such as to do sth let's, etc.) are generally the basic chunks of language, in other words, the basic syntax is equivalent to the language block. After English knowledge gradually enrich, memory by comparing personal and differences, the author in English learning in the early and mid-term met obstacles in the use of the verb "be", also appeared on a kind of and kind of usage of confusion. I was writing and often appear in the "how many people in your family (without are)" and "i'm a kind of hungry (grammar)" this kind of wrong application, the reason for this error, the less amount of reading is the root cause, mainly in the early stage of learning and memory habit, not too much to consider grammar, and after a lot of reading, this kind of grammar combined with 
specific statements, summed up their own understanding of the chunks, such as "how many be", "i'm kind of..." and other chunks, although this kind of lexical chunks in early texts is summarized as the basic grammar, but in English learning is gradually in-depth and many have not been summarized as the usage of grammar or we don't understand, so that writing is prone to error, and to solve this problem is to read a lot, and the accumulation of correct summary Chunks of language, the habit of mastering grammar[4].

Secondly, the accumulation of lexical chunks, improve the efficiency of vocabulary memory retrieval. Mentioned above by reading to strengthen language block should pay attention to the accumulation is sentence language block, avoid writing a common syntax errors, lexical chunks of memory more focus on vocabulary of English usage, lower grade rote memory effect is poor. Therefore, the focus of the vocabulary memory should be how to make a lot of chunks of language, and use them to form a complete sentence and discourse, rather than to explain the independent terms. The English word memory ability and language recognition ability, in vocabulary learning, can not simply reciting, dictation, should appropriate to join match usage, in the appropriate context into their own understanding of the lexical block. For example, "it has been very busy, I haven't stopped! I feel like I've been working 24 / 7. " 24 / 7 generally refers to all the meaning, but also of established by usage in English usage, and the early writing often writing day and night. This is a non habitual expression method, and English idioms are significantly different.

In the end, based on the need of coherence writing, the text is constructed. Paragraph is the final framework for the writing of the pursuit, and the writing of the sentence is different, it should have a complete and clear expression structure. The discourse organization block is used to represent the logical relations between sentences, connecting passages and connecting the whole discourse, such as we described a has a double meaning of events would consider "while, but how this idea, but in the application of English although and but is not used at the same time, and in memory can be recalled in English common syntactic structure and avoid redundant, resulting in semantic expression of repeatability of.

\section{Test results}

The most direct effect of language chunks on English learning is to help improve the accumulation of vocabulary, to form a fluent language expression ability, and to grasp the meaning and coherence of the language. For English writing, it has more practical effect. In the previous paper, the application of lexical chunks has strengthened the sense of the language. In practical writing, the requirements of the language is slightly less than that of the conversation, but more attention is paid to the cohesion of the statement. Chunks at this time play a very important organizational skills, such as college English writing often practice standards, such as Syrian thang, description and argumentative also have their conventional word explicit marking of, chunks of accumulation to accelerated write authors memories of specific style and structure, the writing time recall of words for ratio is reduced and speed up your writing.

Lewis (1993) thinks that language is composed of grammatical meaning, but not by grammatical meaning. These syntactic and lexical chunks form a number of continuous (chunks meaningful), and then form a continuous and coherent discourse (coherent and text). For Chinese students, the memory and understanding of the problem is not serious, but from the brain to be quickly extracted from the more difficult, not to mention the appropriate application of the. Wallace (1982) that the teaching of English vocabulary there are some problems: 1, the use of the search is less than the required vocabulary; 2) the use of inappropriate words; 3) the use of inappropriate words; 4) the use of words do not conform to the habit of. Such problems can be solved by the use of lexical approach, lexical chunks or lexical chunks can be used as prefabricated templates, as a whole unit of storage and output. Especially for primary school students, in the absence of knowledge of the language, the language of the language for the realization of communication is undoubtedly a positive role.

From the above classification of lexical chunks can be seen, the word chunks to a word, large to a sentence, master a word can master a lot of words. At the same time, because chunks of the meaning of the specific context. Therefore, chunks of words is easier to remember and intentional 
memory memory than meaningless memory more effectively, this is proved by research in psychology. Moreover, "it has a higher accuracy. Should the chunk composition between the components of the received dual constraints of syntactic and semantic collocation, in use can also from the memory that is to take "(lidazhi, 2006:34). The sentence framework although there are some changes, but they have very strong regularity, can replace part of the flexibility of the relatively small, it is much smaller than the efforts paid by themselves according to the syntax, vocabulary usage and generate sentences and make the possibility of error is much smaller. On the basis of these chunks, the instant communication is more fluent and the accuracy is higher (and Syder Pawley, 1983)[5].

2) the use of pragmatic, correct register

Language learning, which can avoid the use of words and sentences in the process of language learning, can avoid errors in the use of context. The main reason is that the lexical chunks have a strong pragmatic function, and the formation of the lexical chunks itself contains some kind of context of application. For example, are you how What's your name Let's (let's begin our class e.g. let's; go home together.). These chunks have their own application context, is basically a fixed program.

3) the native language of fluency and selectivity

Pawley \& Syber (1983) presented two puzzle: the mother tongue and the mother tongue. Why do native speakers express and accurately select language materials? They believe that mother tongue learners are mainly based on the accumulation of a large number of programming language, there are many lexical sentence stems (lexicalized). So foreign language learners want to achieve or close to their native language learners to master the language of the program, improve the accuracy and fluency of language use.

4) to solve the contradiction between language learning and language expression

Children learning a foreign language is not the first to start from the rules of learning language. Research shows (Wray, 2002), in first language acquisition and analysis exist at the same time (rule-based system) system and the formulaic system (exemplar-based system), but accounts for the proportion in the constant change. From birth to 20 months or so, the formulaic system occupies the absolute superiority in the children's language system. But from 20 months to 30 months, children's analysis ability was significantly improved. From 8 to 18 years old, grammar analysis ability has been developed to a relatively complete, but the use of language in communication is indispensable. In adulthood, analysis ability and the formulaic system balance, they in the use of language play respective (left red coral, 2005). "For foreign language learners, children aged between 5 and 10 will have the awareness of the use of language to block up and and knowledge; lexical chunks is help them to successful communication, self display, and an important strategy for target groups into" 2005:84. Study even found (Wray, 2002), the vocabulary size of some second language learners may be larger than native speakers, but they use lexical competence but Bibi native speakers to far worse. Therefore, to establish and maintain between formulaic system and analysis system of balance is the key to the success of two language acquisition.

\section{Conclusion}

It would appear, the traditional Chinese way of learning English is actually "stupid", which also leads to students in English writing, on the memory of English extraction ability is poor, and children in English speaking countries in the face of writing, mind first appeared in the habit of saying (or the equivalent in chunks), and then consider the word writing. This way is similar to our primary school, junior high school language learning, writing only need to recall a single vocabulary can be successfully completed simple writing.

\section{References}

[1] Zhuang Zhixiang, Huang Wei, Wang Le. Current situation and Prospect of [J]. foreign language teaching multimedia foreign language teaching in China, 2007 (1). 
[2] Li Xiaoxiang. Stage of College English teaching reform of achievements and thinking [C]. Chengdu: University, Ministry of education, English teaching reform expert tour. 2006. 10.

[3] Wang Xiaolei. Study [J]. Heilongjiang science and technology information of independent research students under the network environment, 2008 (1): 142.

[4] Xiao Liangrong, Yu really. On the opportunities and challenges and [J]. language of computer network technology brings to the teaching of College English, 2002.

[5] Shen Yinzhen. Multi culture and Contemporary English teaching [M]. Hangzhou: Zhejiang University press, 2006, (3): 92. 